\title{
Two center experience of capsule endoscopy in Iran: Report
}

\section{on 101 cases [version 1; peer review: 1 approved, 1 approved}

\section{with reservations]}

\author{
Fariborz Mansour-Ghanaei ${ }^{1}$, Morteza Asasi², Farahnaz Joukar(iD2, \\ Rahmatollah Rafiei ${ }^{3}$, Alireza Mansour-Ghanaei ${ }^{4}$, Ehsan Hajipour-Jafroudi ${ }^{5}$ \\ ${ }^{1}$ Gastrointestinal and Liver Diseases Research Center, Razi Hospital, Guilan University of Medical Sciences, Rasht, Iran \\ ${ }^{2}$ Caspian Digestive Diseases Research Center, Guilan University of Medical Sciences, Rasht, Iran \\ ${ }^{3}$ Department of Internal Medicine, School of Medicine, Islamic Azad University, Najafabad, Isfahan, Iran \\ ${ }^{4}$ Shahid Beheshti University of Medical Sciences, Tehran, Iran \\ ${ }^{5}$ Tehran University of Medical Sciences, Tehran, Iran
}

V1 First published: 27 Sep 2017, 6:1765

https://doi.org/10.12688/f1000research.11288.1

Latest published: 12 Feb 2018, 6:1765

https://doi.org/10.12688/f1000research.11288.2

\section{Abstract}

Background: Capsule endoscopy (CE) is a minimally invasive method for the visual examination of the small intestine, which may be for the evaluation and follow-up of patients with Crohn's disease. It can also be used to look at mucosal inflammation.

Methods: This cross sectional study was used to determine the diagnostic efficacy of the CE system by performing a cross-sectional study of cases from 2011-2014. This study involved a total of 101 Iranian patients who were referred to the gastrointestinal and liver diseases outpatient clinics in Guilan (northern Iran) and in Isfahan (central Iran) for complaints of gastrointestinal problems. For all patients, definitive diagnosis had failed with the use of other diagnostic tools and CE was performed. Descriptive analysis was used. The patient population was represented by men and women equally, and the mean age of the patients was $42.3 \pm 17.2$ years (range: $16-89$ years).

Results: The final diagnoses were: non-specific enteritis (30.6\%), Crohn's disease (20.7\%), ulcers caused by aspirin or non-steroidal antiinflammatory drugs (8.9\%), mucosal erosion (5.9\%) and angioectasia (4.9\%); nearly $10 \%$ of the patients had normal findings. Analysis of the distribution of chief presenting complaints with patients stratified by the final diagnosis of Crohn's disease showed that the most frequently presented chief complaint was abdominal pain $42.9 \%$ and the least frequently presented chief complaint was diarrhea (4.8\%).

Conclusions: Small bowel evaluation by CE was well tolerated and capable of diagnosing Crohn's disease and gastrointestinal bleeding in patients who failed other diagnostic tests.

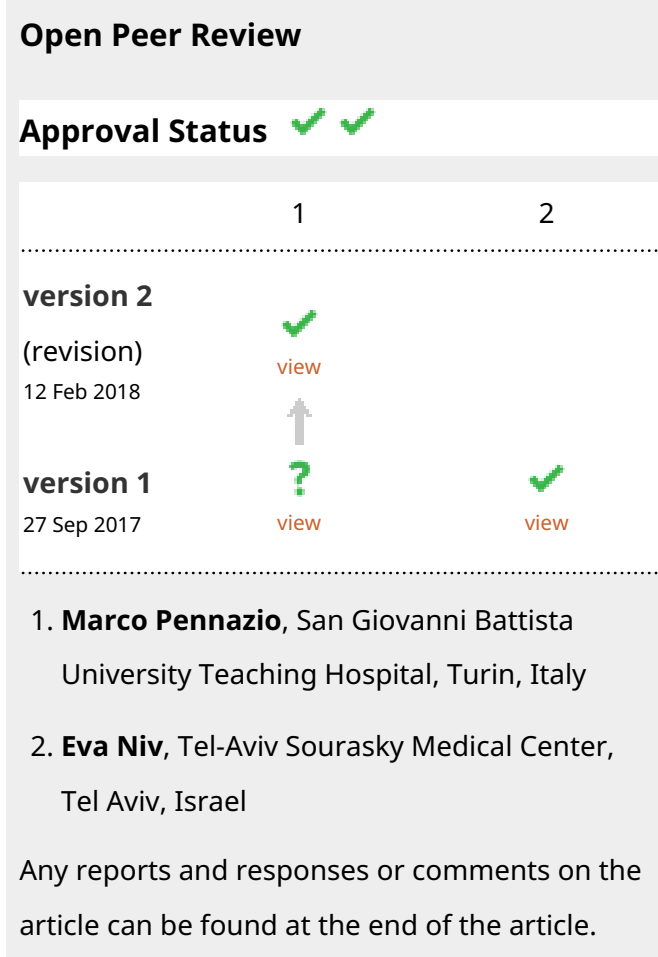

article can be found at the end of the article. 


\section{Keywords}

Capsule Endoscopy, Diagnostic Imaging, Crohn disease, Gastrointestinal Diseases, Double-Balloon Enteroscopy

\section{Corresponding author: Fariborz Mansour-Ghanaei (ghanaie@yahoo.com)}

Competing interests: No competing interests were disclosed.

Grant information: The author(s) declared that no grants were involved in supporting this work.

Copyright: (c) 2017 Mansour-Ghanaei F et al. This is an open access article distributed under the terms of the Creative Commons Attribution License, which permits unrestricted use, distribution, and reproduction in any medium, provided the original work is properly cited. Data associated with the article are available under the terms of the Creative Commons Zero "No rights reserved" data waiver (CCO 1.0 Public domain dedication).

How to cite this article: Mansour-Ghanaei F, Asasi M, Joukar F et al. Two center experience of capsule endoscopy in Iran: Report on 101 cases [version 1; peer review: 1 approved, 1 approved with reservations] F1000Research 2017, 6:1765

https://doi.org/10.12688/f1000research.11288.1

First published: 27 Sep 2017, 6:1765 https://doi.org/10.12688/f1000research.11288.1 


\section{Introduction}

Capsule endoscopy (CE) is a relatively new imaging technique used to visualize, evaluate and diagnose the gastrointestinal tract in a non-invasive manner. While it has proven to be feasible and well tolerated for examination of the small bowel, CE has not yet emerged as an efficacious alternative to traditional endoscopy for the esophagus, stomach, duodenum or colon ${ }^{1}$. Yet, its applications in small bowel continue to evolve and advance.

The recently developed double-balloon enteroscopy system is based upon the classical endoscopic approach of inserting a flexible endoscope, which is covered by a special tube that has two balloons at the end ${ }^{1,2}$. Inflation and deflation of the balloons allow for further advancement into the bowel and more extensive viewing of the mucosa. Although this device is able to facilitate biopsy taking and remedial actions, its application is very time consuming and it may not be feasible to examine the small bowel completely. $\mathrm{CE}$ is another recently developed technique and boasts the distinct advantage of being capable of providing endoscopic evaluation of the small bowel completely ${ }^{3,4}$.

Compared to traditional endoscopy, CE has a higher sensitivity because it allows for examination of otherwise inaccessible areas of the small bowel and facilitates the operator's ability to detect changes and diagnose disease ${ }^{5,6}$. Moreover, the latest advancement in the use of video capsule allows operators to visualize the complete small bowel ${ }^{7}$. During its 4-h to 6-h trek through the small bowel, a capsule will transfer captured images wirelessly to an external receiver that is worn by the patient. These images are of high quality and comparable to those taken by ordinary scopes ${ }^{8,9}$.

CE has particularly high sensitivity and specificity for diagnosis of gastrointestinal lesions. As such, the most popular application of $\mathrm{CE}$ has emerged as determining the causes of gastrointestinal symptoms, such as gastrointestinal bleeding, malabsorption and unspecified abdominal pain; the main diagnoses are small bowel tumors, angiodysplasia and inflammatory diseases, such as Crohn's disease, infectious enteritis, celiac sprue and ulcers caused by use of non-steroidal anti-inflammatory drugs ${ }^{1,2,6}$. Several studies have also shown the utility of CE for diagnosis of celiac disease and its complications ${ }^{10-12}$, and for detection of polyps to screen for Peutz-Jeghers syndrome and familial adenomatous polyposis ${ }^{2,5,7}$. Hence, video CE would represent an alternative option for patients who are unable or unwilling to undergo esophagogastroduodenoscopy ${ }^{13}$. Contraindications to CE include known or suspected bowel obstruction, strictures or fistulas (which have been detected by other clinical imaging or tests prior), cardiac pacemakers, implanted electro-myocardial tools and swallowing disorders ${ }^{14}$.

The OMOM CE System, manufactured by Jinshan Science \& Technology (Group) Co., Ltd (Chongqing, China), provides good quality images and is available at a reasonable price, making it a feasible option for smaller healthcare institutes and/or countries with developing economies ${ }^{15}$. In this study, we performed OMOM CE to diagnose gastrointestinal diseases in adult patients referred to two gastroenterology clinics in the Guilan and
Isfahan provinces of Iran for evaluation of various gastrointestinal complaints between January 2011 and February 2014. The study was performed in order to distinguish the proficiency of OMOM $\mathrm{CE}$ and highlight its importance to other gastroenterologists.

\section{Methods}

This cross-sectional study included patients that were referred to the gastrointestinal and liver disease outpatient clinic of Razi Hospital of the Guilan University of Medical Sciences (GUMS) in Rasht, north of Iran, and Dr Rafiei's gastroenterology clinic in Isfahan (private clinic), with a nonspecific age criteria and symptoms such as gastrointestinal bleeding of unknown origin; abdominal pain; chronic diarrhea; suspected inflammatory bowel disease; iron deficiency anemia; suspected tumors and/or polyps; malabsorption; unintentional weight loss without any diagnosis by other type of conventional evaluation. Patients were denied enrollment if any of the following were present: known or suspected bowel obstruction; strictures or fistulas detected by prior clinical imaging or tests; cardiac pacemakers; implanted electro-myocardial tools; swallowing disorders.

All the examinations were carried out under the following conditions. Iron supplementation was stopped $3 \mathrm{~d}$ before the examination. Consumption of antacids or bismuth components, which are known to coat the camera lens, was discontinued 1d before the examination. Starting at 8 am on the day before the test, the patient was permitted only clear liquids with a light breakfast. The patients consumed one dose (70 g) of polyethylene glycol laxative mixed in $250 \mathrm{~mL}$ water at $4 \mathrm{pm}$ on the day before the test. All the patients were fully fasting starting at $8 \mathrm{pm}$ on the day before the test, with the exception of any critical medications, which were given with sips of water. The procedure was performed at 8 am on the test day.

The following data was collected for each study participant: age; sex; clinical manifestation; chief complaint; preliminary tests performed, including hemoglobin and stool (ova \& parasite, occult blood); final diagnosis with CE.

\section{Data analysis}

The acquired images were reviewed by the two coordinate gastroenterologists. For the descriptive analyses, quantitative variables are expressed as means with standard deviation values; data of analyses of qualitative variables are expressed as frequency and percentages.

\section{Ethical statement}

The Medical Ethics Committee of GUMS (P/3/132) has approved the study design, protocol and informed consent procedure. All measurements were performed based on ethical guidelines of the 1975 Declaration of Helsinki. Informed written consent was obtained from each patient. All patients consented to the study.

\section{Results}

A total of 101 patients were enrolled in the study, based upon the inclusion and exclusion criteria. The study population was represented equally by the two sexes ( $48.5 \%$ male), and the mean age 
of the patients was $42.3 \pm 17.2$ years (range: $16-89$ years). The most frequent chief complaints that led to evaluation by $\mathrm{CE}$ were abdominal pain and anemia, accounting for $40.6 \%$ and $21.8 \%$ of the cases, respectively (Table 1 and Table 2). In patients with Crohn's disease, the most frequent chief complaint was abdominal pain $42.9 \%$ and the least frequent was diarrhea (4.8\%).

When the patients were grouped by sex, Crohn's disease was the most frequent diagnosis in both males $(61.9 \%)$ and females $(38.1 \%)$. When the patients were grouped by age, Crohn's disease was the most frequent diagnosis for both young adults ( $<30$ years: $61.9 \%)$ and middle-aged adults (30-50 years: $28.6 \%)$, and angioectasia was the most frequent in patients $>50$ years in age. Some of the observed results are shown in Figure 1.

\section{Table 1. Chief complaints of patients diagnosed by OMOM capsule endoscopy.}

\begin{tabular}{|l|l|}
\hline Chief compliant & $\boldsymbol{n}(\%)$ \\
\hline Abdominal pain & $41(40.6)$ \\
\hline Diarrhea & $9(8.9)$ \\
\hline Anemia & $22(21.8)$ \\
\hline Occult blood in stool & $9(8.9)$ \\
\hline Abdominal pain + diarrhea & $12(11.9)$ \\
\hline Abdominal pain + anemia & $8(7.9)$ \\
\hline
\end{tabular}

Dataset 1. Data for all the variables collected for each study participant in SPSS and Excel format, i.e. age, sex, gender, clinical manifestation, preliminary tests performed (DX-primary), final diagnose with CE (DX-End)

http://dx.doi.org/10.5256/f1000research.11288.d178638

Table 2. Findings of OMEM capsule endoscopy for the 101 patients in this study.

\begin{tabular}{|l|l|}
\hline Final diagnosis & $\boldsymbol{n}(\%)$ \\
\hline Crohn's disease & $21(20.7)$ \\
\hline Angioectasia & $5(4.9)$ \\
\hline Non-specific enteritis & $31(30.6)$ \\
\hline Ulcers caused by aspirin or NSAIDs & $9(8.9)$ \\
\hline Small intestine tumors & $2(1.9)$ \\
\hline Gastropathy & $12(11.8)$ \\
\hline Celiac disease & $1(1.0)$ \\
\hline Tapeworm & $1(1.0)$ \\
\hline Small intestine polyps & $2(1.9)$ \\
\hline Small intestine mucosal erosion & $6(5.9)$ \\
\hline Gastric polyp & $1(1.0)$ \\
\hline No disease & $10(9.9)$ \\
\hline
\end{tabular}

NSAIDs: non-steroidal anti-inflammatory drugs.
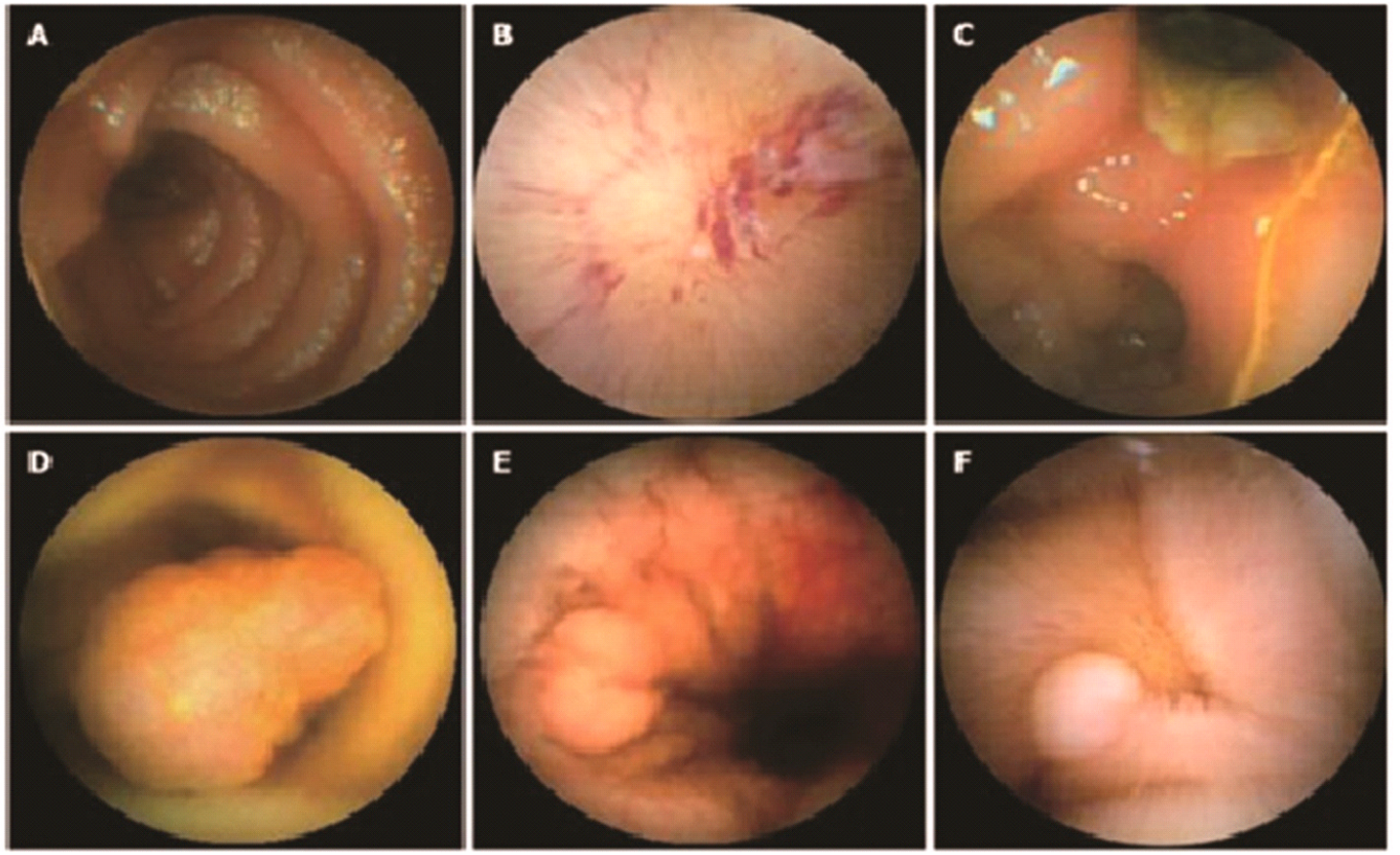

Figure 1. Small bowel images captured by OMOM capsule endoscopy. (A) Normal small bowel mucosa; (B) Angioectasia; (C) Ulcer; (D) Tumor; (E) Crohn's disease; (F) Polyp. 


\section{Discussion}

CE was first described by its inventor Gavriel Iddan in 1981, and since has become a commonly applied clinical tool for evaluation of gastrointestinal disorders and diagnosis of gastrointestinal diseases. The current study, however, represents the first of its kind to be carried out in hospitals and patients in Iran. A similar study was conducted previously in New York in the United States ${ }^{16}$, but the patient population was slightly skewed towards the female sex (57\% vs $48 \%$ in our study) and the mean age of patients was higher (61 years vs 43 years in our study). Moreover, the previous study focused on patients who had been referred for gastrointestinal bleeding, whereas our patients were referred for a variety of common gastrointestinal complaints.

The OMOM CE system is used throughout China and in many European and other Asian countries. The OMOM capsule is slightly larger than another popular small bowel capsules, such as the PillCam capsule, but our patients experienced no problems with its use. Similar to the PillCam capsule's battery, the OMOM capsule's battery can last $8 \mathrm{~h}$. Two exclusive features of CE, as compared to traditional endoscopy, are its ability for real-time imaging and recording of information ${ }^{8}$. A new type of capsule, with a smaller size and a new shape, has just recently been developed and released to market by the OMOM manufacturer in China, and this will likely advance the use of CE even further.

The current study investigated patients treated with the older OMOM capsule, exclusively. The most frequent chief complaints that led to $\mathrm{CE}$ evaluation were abdominal pain, either in isolation or accompanied by diarrhea; this complaint distribution fits with the frequent diagnosis of Crohn's disease $(28.6 \%$ of patients that had abdominal pain and diarrhea, and $42.9 \%$ that had abdominal pain in isolation). These findings were different from those in the study by Ruuska et al. ${ }^{17}$, where abdominal pain in isolation was the most frequent chief complaint (42\%), followed by diarrhea (17\%) and weight loss (5\%). Similarly, a study Mohan et al. ${ }^{18}$ examined the role of $\mathrm{CE}$ in evaluation of patients with suspected small bowel bleeding and identified the most frequent chief complaint as abdominal pain $(43 \%)$ and a small portion of patients with the chief complaint of weight loss $(6 \%)$.

CE is commonly used to evaluate cases of occult gastrointestinal bleeding, such as that which underlies iron deficiency anemia, Crohn's disease and small intestinal tumors; its utility has also been proven for evaluating polyposis syndromes and refractory malabsorptive syndromes, such as celiac disease ${ }^{6}$. According to the literature, the most common uses of OMOM CE in China involve patients with occult gastrointestinal bleeding and who present with complaints of abdominal pain and chronic diarrhea ${ }^{6}$. CE findings of inflammation in the small bowel are indicative of inflammatory bowel disease, and help clinicians to diagnose Crohn's and other inflammation-related disorders ${ }^{19}$. A 2003 study of the technical performance and efficiency of CE reported by Mylonaki et al. ${ }^{20}$, which investigated 50 patients with gastrointestinal bleeding undiagnosed by colonoscopy and gastroscopy, found the diagnosis rate of occult gastrointestinal bleeding was $\sim 43 \%$.

Incidence of Crohn's disease is increasing among the Chinese ${ }^{17}$ and Iran is facing a similar situation ${ }^{21}$. Zheng and colleagues ${ }^{22}$ warned that, while Crohn's disease incidence and prevalence rates in China are still lower than the rates reported from Western countries and even Asian industrialized countries, they are increasing rapidly. Previous studies, including one in 2007 by Fidder et al. ${ }^{23}$ have recommended the use of CE to diagnose Crohn's disease, based upon the reported evidence of its ability to detect the disease condition in a very small percentage of patients (0 to $4 \%$ ).

In our study, two patients were diagnosed with small intestinal tumors. The 2010 study by Jung Wan Han et al. ${ }^{7}$ indicated that CE was capable of detecting particularly challenging or otherwise undetectable tumors in the small bowel. Without CE, many of these tumors may only become detectable by the other available technologies at the later or last stages of cancer, when therapies are less efficacious or feasible. Those authors also reported that $\mathrm{CE}$ has sufficiently higher diagnostic yield and sensitivity for definitive small bowel tumors.

In the present study, one patient was diagnosed with a tapeworm by CE. Several other studies have reported hookworm detection by $\mathrm{CE}$, including a report of 26 Chinese patients with occult gastrointestinal bleeding published by Liao et al. ${ }^{17}$, in which $3.4 \%$ of the patients were diagnosed with hookworm. Another 6 case reports, 4 of which were from Asian countries, showed hookworm as the etiology of gastrointestinal bleeding, as diagnosed by $\mathrm{CE}^{19,24-28}$.

\section{Conclusions}

Medical science is continually looking for ways to eliminate any aggressive (invasive and/or risky) methods of evaluating the body, and the CE method is an excellent way to detect small bowel diseases when traditional endoscopy cannot detect the problems. The OMOM CE system in particular, is a valuable device for small bowel evaluation because of the small size of its capsule, high-resolution images and low price, which supports its use in healthcare settings across the globe and in the diagnosis of common gastrointestinal complaints, especially Crohn's disease.

\section{Data availability}

Dataset 1: Data for all the variables collected for each study participant in SPSS and Excel format, i.e. age, sex, gender, clinical manifestation, preliminary tests performed (DX-primary), final diagnose with CE (DX-End). doi, 10.5256/f1000research.11288. d178638 29 


\section{Author contributions}

Mansour-Ghanaei $\mathrm{F}$ and Asasi $\mathrm{M}$ designed the study; Joukar F supervised the surgical procedures; Mansour-Ghanaei F, Asasi M, Rafiei R, Mansour-Ghanaei A and Hajipour-Jafroudi E collected the data; Joukar F analyzed the data; Mansour-Ghanaei F and Joukar F wrote the manuscript; all authors approved the final version.

\section{Competing interests}

No competing interests were disclosed.
Grant information

The author(s) declared that no grants were involved in supporting this work.

\section{Acknowledgments}

We would like to thank all members of Gastrointestinal \& Liver Diseases Research Center (GLDRC). Also we would like to thank all the hospitals staff that assisted us in this study.
1. Rao SS, Kuo B, McCallum RW, et al:: Investigation of colonic and whole-gut transit with wireless motility capsule and radiopaque markers in constipation. Clin Gastroenterol Hepatol. 2009; 7(5): 537-44.

PubMed Abstract | Publisher Full Text

2. Tran K, Brun R, Kuo B: Evaluation of regional and whole gut motility using the wireless motility capsule: relevance in clinical practice. Therap Adv Gastroenterol. 2012; 5(4): 249-60.

PubMed Abstract | Publisher Full Text | Free Full Text

3. Biagi F, Rondonotti E, Campanella J, et al.: Video capsule endoscopy and histology for small-bowel mucosa evaluation: a comparison performed by blinded observers. Clin Gastroenterol Hepatol. 2006; 4(8): 998-1003. PubMed Abstract | Publisher Full Text

4. Pennazio M: Capsule endoscopy: where are we after 6 years of clinical use? Dig Liver Dis. 2006; 38(12): 867-78. PubMed Abstract | Publisher Full Tex

5. Matas JL, Asteinza M, Loscos JM, et al.: Diagnostic yield and safety of capsule endoscopy. Rev Esp Enferm Dig. 2006; 98(9): 666-73. PubMed Abstract | Publisher Full Text

6. Petroniene R, Dubcenco E, Baker JP, et al:: Given capsule endoscopy in celiac disease: evaluation of diagnostic accuracy and interobserver agreement. Am J Gastroenterol. 2005; 100(3): 685-94.

PubMed Abstract | Publisher Full Text

7. Han JW, Hong SN, Jang HJ, et al:: Clinical Efficacy of Various Diagnostic Tests for Small Bowel Tumors and Clinical Features of Tumors Missed by Capsule Endoscopy. Gastroenterol Res Pract. 2015; 2015; 623208 PubMed Abstract | Publisher Full Text | Free Full Text

8. Delvaux M, Gay G: Capsule endoscopy: technique and indications. Best Pract Res Clin Gastroenterol. 2008; 22(5): 813-37. PubMed Abstract | Publisher Full Text

9. Leighton JA, Shiff AD: Capsule endoscopy: ways to avoid missing lesions and to optimize diagnostic yield. Tech Gastrointest Endosc. 2006; 8(4): 169-74. Publisher Full Text

10. Akin E, Ersoy O: Capsule endoscopy in celiac disease. Gastroenterol Res Pract 2011; 2012; 676073.

PubMed Abstract | Publisher Full Text | Free Full Text

11. Chang MS, Rubin M, Lewis SK, et al.: Diagnosing celiac disease by video capsule endoscopy (VCE) when esophagogastroduodenoscopy (EGD) and biopsy is unable to provide a diagnosis: a case series. BMC Gastroenterol. 2012; 12(1): 90.

PubMed Abstract | Publisher Full Text | Free Full Text

12. Rubio-Tapia A, Hill ID, Kelly CP, et al:: ACG clinical guidelines: diagnosis and management of celiac disease. Am J Gastroenterol. 2013; 108(5): 656-76; quiz 677. PubMed Abstract | Publisher Full Text | Free Full Text

13. Spada C, Riccioni ME, Urgesi R, et al:: Capsule endoscopy in celiac disease. World J Gastroenterol. 2008; 14(26): 4146-51. PubMed Abstract | Free Full Text

14. Niemenmaa $\mathrm{H}$, Mäkelä $\mathrm{T}$, Jussila $\mathrm{A}$, et al:: The diagnostic value of video capsule endoscopy. Eur J Intern Med. 2010; 21(5): 383-5. PubMed Abstract | Publisher Full Text

15. Li CY, Zhang BL, Chen CX, et al: OMOM capsule endoscopy in diagnosis of small bowel disease. J Zhejiang Univ Sci B. 2008; 9(11): 857-62. PubMed Abstract | Publisher Full Text | Free Full Text

16. Schnoll-Sussman F, Kulkarni K: Risks of capsule endoscopy. Tech Gastrointest Endosc. 2008; 10(1): 25-30.

Publisher Full Text

17. Ruuska $T$, Vaajalahti $\mathrm{P}$, Arajärvi $\mathrm{P}$, et al:: Prospective evaluation of upper gastrointestinal mucosal lesions in children with ulcerative colitis and Crohn's disease. J Pediatr Gastroenterol Nutr. 1994; 19(2): 181-6. PubMed Abstract | Publisher Full Text

18. Khadka M, Tao X, Chen DR, et al:: One year experience of OMOM Capsule Endoscopy for suspected intestine lesions. Journal of Nobel Medical College. 2011; 1(1): 27-39. Publisher Full Text

19. Li ZS, Liao Z, Ye P, et al.: Dancing hookworm in the small bowel detected by capsule endoscopy: a synthesized video. Endoscopy. 2007; 39(Suppl 1): E97. PubMed Abstract | Publisher Full Text

20. Mylonaki M, Fritscher-Ravens A, Swain P: Wireless capsule endoscopy: a comparison with push enteroscopy in patients with gastroscopy and colonoscopy negative gastrointestinal bleeding. Gut. 2003; 52(8): 1122-6. PubMed Abstract | Publisher Full Text | Free Full Text

21. Mansour-Ghanaei F, Haghkerdar M, Joukar F, et al:: Epidemiologic Features of Inflammatory Bowel Disease in Guilan Province, North of Iran, During 2002-2012. Middle East J Dig Dis. 2015; 7(2): 69-74. PubMed Abstract | Free Full Text

22. Zheng JJ, Zhu XS, Huangfu Z, et al.: Crohn's disease in mainland China: a systematic analysis of $\mathbf{5 0}$ years of research. Chin J Dig Dis. 2005; 6(4): 175-81. PubMed Abstract | Publisher Full Text

23. Fidder $\mathrm{HH}$, Nadler M, Lahat A, et al.: The utility of capsule endoscopy in the diagnosis of Crohn's disease based on patient's symptoms. J Clin Gastroenterol. 2007; 41(4): 384-7. PubMed Abstract | Publisher Full Text

24. Chao CC, Ray ML: Education and imaging. Gastrointestinal: Hookworm diagnosed by capsule endoscopy. J Gastroenterol Hepatol. 2006; 21(11): 1754 PubMed Abstract | Publisher Full Text

25. Chen TH, Chen TY, Shyu LY, et al:: Hookworm infestation diagnosed by capsule endoscopy (with video). Gastrointest Endosc. 2006; 64(2): 277-8. PubMed Abstract | Publisher Full Text

26. Croese J, Speare R: Intestinal allergy expels hookworms: seeing is believing Trends Parasitol. 2006; 22(12): 547-50. PubMed Abstract | Publisher Full Text

27. Morales CP, Ferrer G, Zuckerman MJ: Hookworm detected by capsule endoscopy. Gastrointest Endosc. 2005; 62(5): 782-3; discussion 783. PubMed Abstract | Publisher Full Text

28. Wu IC, Lu CY, Wu DC: Acute hookworm infection revealed by capsule endoscopy. Endoscopy. 2007; 39(Suppl 1): E306. PubMed Abstract | Publisher Full Text

29. Mansour-Ghanaei F, Asasi M, Joukar F, et al.: Dataset 1 in: Two center experience of capsule endoscopy in Iran: Report on 101 cases. F1000Research. 2017.

Data Source 


\section{Open Peer Review}

\section{Current Peer Review Status:}

\section{Version 1}

Reviewer Report 16 January 2018

https://doi.org/10.5256/f1000research.12178.r29793

(C) 2018 Niv E. This is an open access peer review report distributed under the terms of the Creative Commons Attribution License, which permits unrestricted use, distribution, and reproduction in any medium, provided the original work is properly cited.

\section{Eva Niv}

Capsule Endoscopy Unit, Department of Gastroenterology, Tel-Aviv Sourasky Medical Center, Tel Aviv, Israel

The paper is well-written and presents interesting data regarding the results of capsule endoscopy in Iran. It presents data about the prevalence of different small bowel pathologies in Iran population. I support the publication of this paper. For my opinion, there is no need in changes, besides minor language corrections.

Is the work clearly and accurately presented and does it cite the current literature? Yes

Is the study design appropriate and is the work technically sound? Yes

Are sufficient details of methods and analysis provided to allow replication by others? Yes

If applicable, is the statistical analysis and its interpretation appropriate?

Yes

Are all the source data underlying the results available to ensure full reproducibility? Yes

Are the conclusions drawn adequately supported by the results?

Yes

Competing Interests: No competing interests were disclosed.

Reviewer Expertise: Gastroenterology, small bowel diseases, Crohn's disease, 15 years of 
experience in the field of capsule endoscopy

I confirm that I have read this submission and believe that I have an appropriate level of expertise to confirm that it is of an acceptable scientific standard.

Reviewer Report 12 December 2017

https://doi.org/10.5256/f1000research.12178.r28927

(C) 2017 Pennazio M. This is an open access peer review report distributed under the terms of the Creative Commons Attribution License, which permits unrestricted use, distribution, and reproduction in any medium, provided the original work is properly cited.

\section{Marco Pennazio}

Gastroenterology and Hepatology Department of Medicine, San Giovanni Battista University Teaching Hospital, Turin, 10123, Italy

This is a descriptive report on the use of capsule endoscopy for the investigation of a quite heterogenoeous population.

I suggest to better specify:

1. In patients with Crohn's disease: was this diagnosis suspected before CE or Crohn's disease was already Known before CE

2. specify if, in patients with known Crohn's disease before $C E$, the subsequent endoscopic examination turned out to be negative

3. if the diagnosis of Crohn's disease was only suspected before CE, please specify how the diagnosis was made on the basis of the capsule findings

4. did the authors observed any complication after CE (ie capsule retention)?

5. in the discussion please underline that patients with known Crohn's disease are at increased risk of capsule retention and therefore every effort (patency capsule; MRE before CE) should be made in order to minimize this risk.

6. with reference to this last point please quote: Pennazio M, Spada C, Eliakim Small-bowel capsule endoscopy and device-assisted enteroscopy for diagnosis and treatment of smallbowel disorders: European Society of Gastrointestinal Endoscopy (ESGE) Clinical Guideline. Endoscopy 2015;47(4):352-76.

Is the work clearly and accurately presented and does it cite the current literature? Partly

Is the study design appropriate and is the work technically sound?

Partly 
Are sufficient details of methods and analysis provided to allow replication by others? Partly

If applicable, is the statistical analysis and its interpretation appropriate?

Not applicable

Are all the source data underlying the results available to ensure full reproducibility? Partly

Are the conclusions drawn adequately supported by the results?

Partly

Competing Interests: No competing interests were disclosed.

I confirm that I have read this submission and believe that I have an appropriate level of expertise to confirm that it is of an acceptable scientific standard, however I have significant reservations, as outlined above.

Author Response 15 Jan 2018

Farahnaz Joukar, Guilan University of Medical Sciences, Rasht, Iran

Thank you very much for the insightful review comments. Here are the related point-bypoint replies according to the comments.

1. The diagnosis of questionable patients with Crohn's disease before capsule endoscopy was based on existence of abdominal pain, anemia and the physician clinical suspicious

2. The capsule endoscopy findings of Crohn's disease among patients were after exclusion of NSAID consumption and diagnosis of parasitic infections. Furthermore, the patient's treatment response was considered as the diagnosis confirmation.

3. After capsule endoscopy no side effects were reported.

4. It is added in the text as "Although capsule retention is one of the reported side effects of capsule endoscopy in patients with Crohn's disease ${ }^{30}$, in our study this issue was not observed".

5. In the last part of the methods section we have added "Also, small bowel series were conducted for all patients".

Competing Interests: None declared 
The benefits of publishing with F1000Research:

- Your article is published within days, with no editorial bias

- You can publish traditional articles, null/negative results, case reports, data notes and more

- The peer review process is transparent and collaborative

- Your article is indexed in PubMed after passing peer review

- Dedicated customer support at every stage

For pre-submission enquiries, contact research@f1000.com 